\title{
Acute disseminated encephalomyelitis-like presentation after an inactivated coronavirus vaccine
}

\author{
Gulay Ozgen Kenangil ${ }^{1}$ Buse Cagla Ari ${ }^{1}$ (1) $\cdot$ Cagan $_{\text {Guler }}{ }^{1} \cdot$ Mustafa Kemal Demir $^{2}$
}

Received: 2 April 2021 / Accepted: 30 April 2021 / Published online: 20 May 2021

(c) Belgian Neurological Society 2021

\section{Dear Editor,}

We are facing a global pandemic with Severe Acute Respiratory Syndrome Coronavirus 2 (SARS-CoV-2) infection, according to the definition of the World Health Organization (WHO), since 11 March 2020. There are several types of vaccines being used all over the world against SARS-CoV-2 infection. Turkey has already started the vaccination program with an inactivated SARS-CoV-2 vaccine of Sinovac (Vero Cells, Beijing Institute of Biological Products Co., Ltd., Beijing, China), and an mRNA-based COVID-19 vaccine of Pfizer-BioNTech, Germany. Although studies to date indicate the vaccines for COVID-19 have an excellent safety profile, severe complications may occur, as seen with previous virus vaccines [1]. Vaccines are one of the etiological factors of Acute Disseminated Encephalomyelitis (ADEM), an immune-mediated demyelinating disease of the brain and the spinal cord, although it is extremely rare [2, 3].

Herein, we report a 46-year-old woman who presented to the hospital, and reported that she had experienced the first tonic-clonic seizure of her life 4 days ago. She denied any prior illness other than Hashimoto's thyroiditis for which she was taking levothyroxine $100 \mathrm{mg} /$ daily. She received her second dose of SARS-CoV-2 vaccination with Sinovac 1 month before the seizure. She is a smoker, but did not use illicit drugs or heavy alcohol. She did not have fever, cough, malaise, or headache. Her neurological examination was entirely normal. She had cranial magnetic resonance imaging (MRI) to investigate the etiological cause of her seizure. There were scattered hyperintense lesions in the left thalamus, bilateral corona radiata, left diencephalon, and

Buse Cagla Ari

juvelia@gmail.com

1 Bahcesehir University Faculty of Medicine, Department of Neurology, Istanbul, Turkey

2 Bahcesehir University Faculty of Medicine, Department of Radiology, Istanbul, Turkey right parietal cortex on T2 and FLAIR sequences on MRI. Some of these lesions showed mild restricted diffusion on DWI (Fig. 1). We detected no enhancement on post-gadolinium contrast-enhanced images. MRI spectroscopy was performed to rule out a diffuse glioma, and did not show choline (Cho) elevation. Her serum thyroid-stimulating hormone (TSH) level was $0.21 \mathrm{IU} / \mathrm{ml}(0.35-4.949)$, and free triiodothyronine was 2.4 (normal). The findings of other laboratory tests, including extensive cerebrospinal fluid (CSF) studies, were normal except ANA (1/100) and Anti-SOX1 antibody positivity. In particular, CSF was acellular with normal protein content $(45 \mathrm{~mm} / \mathrm{dL})$, an $\mathrm{IgG}$ index of 0.64 and no oligoclonal bands. CMV IgM and IgG, EBV IgG, VZV IgM, HSV-1 IgM, and IgG antibodies were all negative in CSF. Anti-double-stranded DNA and extractable nuclear antigen (ENA) panel, anti-aquaporin-4 and anti-myelin oligodendrocyte (MOG) antibodies were negative in serum. Her C 3 and C4 levels were normal. She denied any rash or artritis or sun sensitivity in her history. She had a negative nasal swab test for SARS-CoV-2, and her SARS-CoV-2 IgG antibody was $877 \mathrm{AU} / \mathrm{mL}$. Thorax CT, and whole-body positron emission tomography-computed tomography (PET-CT) were unremarkable. She was put on a $1 \mathrm{~g} /$ day steroid treatment for 7 days, and her Euthytrox (Levothyroxine Sodium tablets, USP) $100 \mathrm{mg} /$ daily treatment was reduced to $75 \mathrm{mg} /$ daily accordingly. After 2 weeks of treatment, she had a control MRI, in which the lesions showed prominent prolonged restricted diffusion without any change in the lesions' size (Fig. 2). On her clinical follow-up, she did not have any new signs, symptoms, or seizures.

Since the patient's clinical findings do not fulfill the clinical criteria for the diagnosis of ADEM due to the absence of encephalopathy, we prefer to define this clinical situation and MRI findings as an ADEM-like presentation after an inactivated coronavirus vaccine. After reviewing the literature, we have found only one case of ADEM after vaccination for SARS-CoV-2 [4]. The cases had symptoms like 

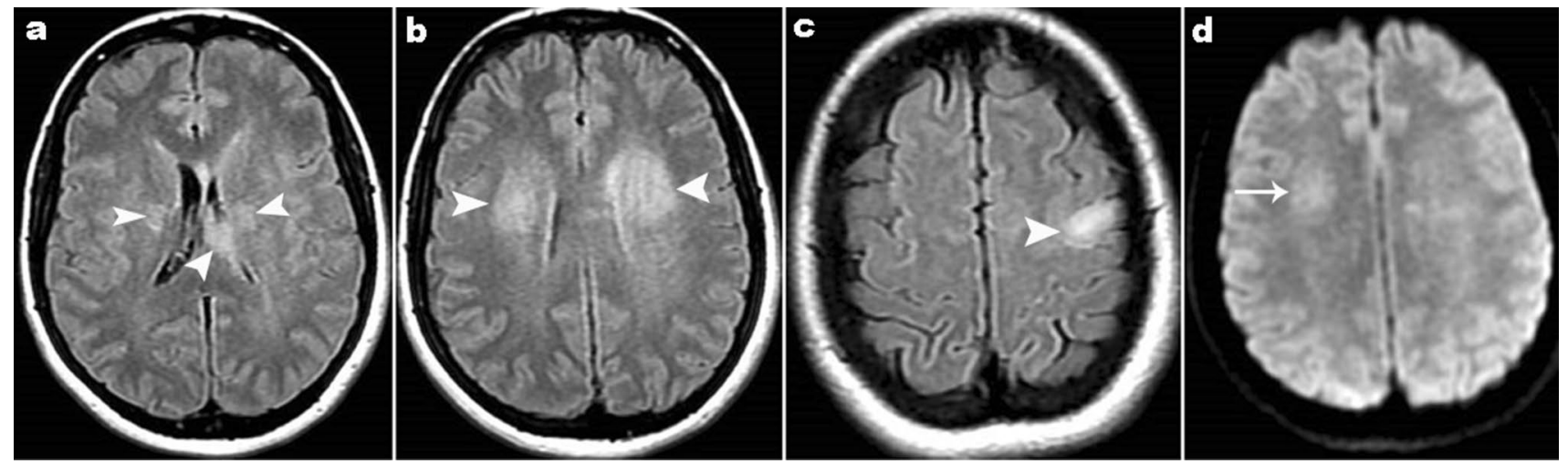

Fig. 1 a-c Axial fluid-attenuated inversion recovery (FLAIR) MRI of the brain demonstrates multifocal areas of hyperintensity in both cerebral hemispheres involving bilateral corona radiata, bilateral cen-

trum semiovale, corpus callosum, and left frontal cortical gray matter (arrowheads). d The diffusion-weighted image shows the lesion in the left centrum semiovale to be mildly hyperintense (arrow)

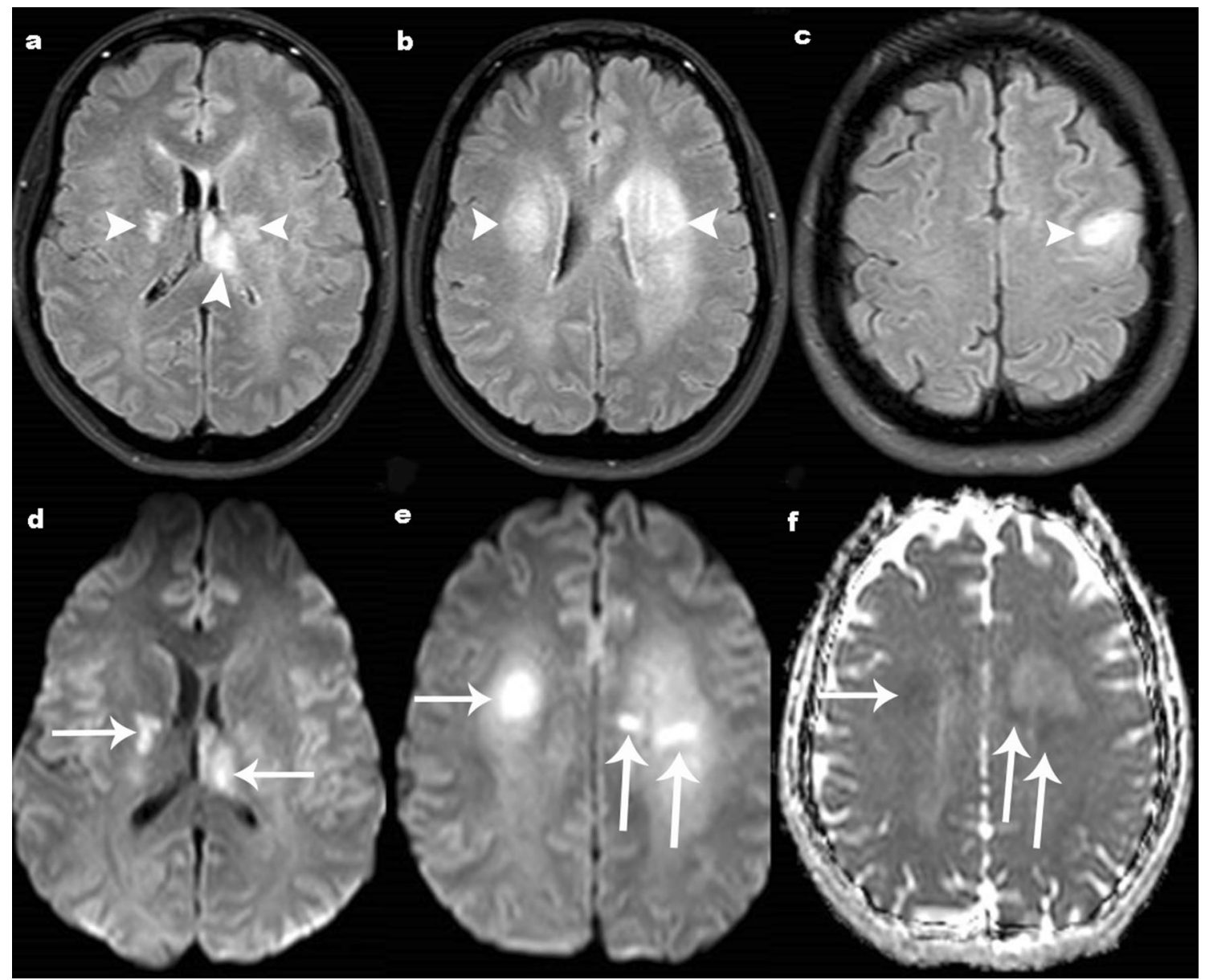

Fig. 2 a-c Repeated MRI after 2 weeks of treatment. There is no significant change on FLAIR images (arrowheads). However, some of the lesions show prominent hyperintensity on DWIs (arrows) (a,

headaches, muscle stiffness, extremity weakness, fever, and more restricted lesions in the brain, but not a seizure. b), and hypointensity on corresponding apparent diffusion coefficient (ADC) map due to increased and prolonged restricted diffusion (arrows) (c)

In conclusion, an ADEM-like presentation similar to the presented case has not been reported following SARSCoV-2 vaccination before. Moreover, this case informs us 
that, even though it is an extremely rare condition, clinicians should be aware of the possible atypical neurological complications in patients receiving this vaccine to iniciate the appropriate treatment.

\section{Declarations}

Conflict of interest The authors declare that they have no competing interests.

Ethical approval This article does not contain any studies with human participants performed by any of the authors.

Informed consent Informed consent was obtained from the patient. illustrative case. J Clin Neurosci 15:1315-1322. https://doi.org/ 10.1016/j.jocn.2008.05.002

2. Torisu H, Okada K (2019) Vaccination-associated acute disseminated encephalomyelitis. Vaccine 37:1126-1129. https://doi.org/ 10.1016/j.vaccine.2019.01.021

3. Pohl D, Alper G, Van Haren K, Kornberg AJ, Lucchinetti CF, Tenembaum S et al (2016) Acute disseminated encephalomyelitis: updates on an inflammatory CNS syndrome. Neurology 87:S3845. https://doi.org/10.1212/WNL.0000000000002825

4. Cao L, Ren L (2021) Acute disseminated encephalomyelitis after severe acute respiratory syndrome coronavirus 2 vaccination: a case report. Acta Neurol Belg 1:1-3. https://doi.org/10.1007/ s13760-021-01608-2

Publisher's Note Springer Nature remains neutral with regard to jurisdictional claims in published maps and institutional affiliations.

\section{References}

1. Huynh W, Cordato DJ, Kehdi E, Masters LT, Dedousis C (2008) Post-vaccination encephalomyelitis: literature review and 